Article

\title{
The Role of Distinctive Sphingolipids in the Inflammatory and Apoptotic Effects of Electronegative LDL on Monocytes
}

\author{
Núria Puig ${ }^{1,2}$, Montserrat Estruch ${ }^{1}$, Lei Jin ${ }^{1}$, Jose Luis Sanchez-Quesada ${ }^{1,3} \mathbb{D}$ and \\ Sonia Benitez ${ }^{1, *}$ \\ 1 Cardiovascular Biochemistry, Biomedical Research Institute Sant Pau (IIB-Sant Pau), Barcelona, Spain. \\ C/Sant Quinti 77-79, 08041 Barcelona, Spain \\ 2 Department of Biochemistry and Molecular Biology, Faculty of Medicine, Building M, \\ Universitat Autònoma de Barcelona (UAB), Cerdanyola del Vallès, 08193 Barcelona, Spain \\ 3 CIBER of Diabetes and Metabolic Diseases (CIBERDEM), 28029 Madrid, Spain \\ * Correspondence: sbenitez@santpau.cat; Tel.: +34-935-537-595; Fax: +34-935-537-589
}

Received: 3 July 2019; Accepted: 20 July 2019; Published: 24 July 2019

\begin{abstract}
Electronegative low-density lipoprotein (LDL $(-))$ is a minor LDL subfraction that is present in blood with inflammatory and apoptotic effects. We aimed to evaluate the role of sphingolipids ceramide (Cer), sphingosine (Sph), and sphingosine-1-phosphate (S1P) in the LDL(-)-induced effect on monocytes. Total LDL was subfractioned into native LDL and LDL(-) by anion-exchange chromatography and their sphingolipid content evaluated by mass spectrometry. LDL subfractions were incubated with monocytes in the presence or absence of enzyme inhibitors: chlorpromazine (CPZ), D-erythro-2-(N-myristoyl amino)-1-phenyl-1-propanol (MAPP), and N,N-dimethylsphingosine (DMS), which inhibit Cer, Sph, and S1P generation, respectively. After incubation, we evaluated cytokine release by enzyme-linked immunosorbent assay (ELISA) and apoptosis by flow cytometry. LDL(-) had an increased content in Cer and Sph compared to LDL(+). LDL(-)-induced cytokine release from cultured monocytes was inhibited by CPZ and MAPP, whereas DMS had no effect. LDL(-) promoted monocyte apoptosis, which was inhibited by CPZ, but increased with the addition of DMS. LDL enriched with Sph increased cytokine release in monocytes, and when enriched with Cer, reproduced both the apoptotic and inflammatory effects of LDL( - ). These observations indicate that Cer content contributes to the inflammatory and apoptotic effects of LDL(-) on monocytes, whereas Sph plays a more important role in LDL(-)-induced inflammation, and S1P counteracts apoptosis.
\end{abstract}

Keywords: electronegative LDL; ceramide; sphingosine; inflammation; apoptosis; monocytes

\section{Introduction}

Sphingolipids have an essential structural function in cell membranes. In addition, these lipid metabolites are emerging as important intracellular and intercellular signaling molecules that are involved in the progress of inflammatory diseases and atherosclerosis [1]. These bioactive lipids are present in atherosclerostic plaque, where they play inflammatory and apoptotic roles [2]. Sphingolipid metabolism is complex and highly interconnected, and each step is controlled by specific enzymes. The core component of sphingolipid metabolism is ceramide (Cer), which is generated by the hydrolysis of sphingomyelin (SM) by sphingomyelinase activity (SMase), and can be hydrolyzed by ceramidase (CDase) activity, yielding sphingosine (Sph). Sph can subsequently be phosphorylated by S1P kinase to generate sphingosine-1-phosphate (S1P) [3].

Cer is a widely studied molecule that regulates cell differentiation, inflammation, and apoptosis [4]. Its plasma concentration is elevated in patients who are at high cardiovascular risk [5,6]. The role of 
Sph in atherosclerosis and inflammation is not clearly established, and its biological effects have been reported to overlap with those promoted by Cer [7]. Sph has been shown to promote inflammatory response [8] and apoptosis [9], although it has also been reported to induce an anti-apoptotic effect [10]. $\mathrm{S} 1 \mathrm{P}$ is a pleiotropic bioactive metabolite, and is an important intercellular signaling molecule recognized by specific cell receptors whose subtype determines the effect on cells [11]. S1P is reported to promote cell proliferation, survival, and migration [12-14]. Regarding inflammation, some studies have described how it promotes inflammation [15-17], whereas others have reported an anti-inflammatory action, mainly when it is bound to its major carrier, high-density lipoprotein (HDL) [18-20]. Although LDL may contain low quantities of S1P, it mainly carries Cer and Sph [21]. Some studies have shown that LDL with an increased Cer content induces inflammation [22,23], whereas the effect of an increased content of Sph and S1P in LDL is unknown.

Electronegative low-density lipoprotein $(\operatorname{LDL}(-))$ is a minor LDL subfraction found in blood circulation that has various atherogenic effects, such as the induction of inflammation in cultured cells. It differs from native LDL $(\mathrm{LDL}(+))$ in several physicochemical properties [24], including the presence of phospholipolytic activities. Indeed, LDL(-) has a fivefold to sixfold increased concentration of the enzyme platelet-activating factor acetylhydrolase (PAF-AH) compared with LDL(+) [25]. In addition, a phospholipase C (PLC)-like activity is believed to be associated with LDL(-), having a high avidity to degrade lysophosphatidylcholine (LPC) by lysoPLC activity and sphingomyelin (SM) by SMase activity [26]. These enzymatic activities seem to be involved in the increased concentrations in LDL(-) of Cer and non-esterified fatty acid (NEFA), which are responsible for LDL(-)-induced cytokine release in monocytes $[22,27,28]$. However, other compounds, such as those resulting from Cer hydrolysis-Sph and S1P - could also be increased in LDL(-) and be involved, not only in the inflammatory effect of LDL(-), but also in a putative apoptotic effect on monocytes.

LDL(-) exerts an apoptotic effect in macrophages [29] and in endothelial cells [30], being attributed in the latter to the increased Cer content in LDL(-) [30]. In fact, Cer and NEFA are inductors of apoptosis [31,32], and an increased content of these molecules confers apoptotic properties on LDL [31,33,34]. Moreover, modified LDL can promote apoptosis by increasing the Cer content in cells [31,35].

In the current study, we sought to determine the specific involvement of the sphingolipids Cer, Sph, and S1P in the inflammatory and apoptotic effects of LDL(-) in human monocytes isolated from plasma. For this purpose, we used specific inhibitors of SMase, CDase, and Sph kinase activities, as summarized in the Methods section.

\section{Material and Methods}

\subsection{LDL Isolation and Modification}

Plasma samples from healthy normolipemic subjects (total cholesterol $<5.2 \mathrm{mmol} / \mathrm{L}$, triglyceride $<1 \mathrm{mmol} / \mathrm{L})$ were obtained in EDTA-containing Vacutainer tubes. All the subjects gave their written informed consent, and the study was conducted after approval from the Institutional Ethics Committee of the Hospital Sant Pau (IIBSP-APO-2013-105, 25 February 2015). All the LDL preparations were performed in conditions that prevented oxidation and endotoxin contamination [36]. LDL (1.019-1.050 kg/L) was isolated from plasma by sequential flotation ultracentrifugation at $4{ }^{\circ} \mathrm{C}$. In some experiments, LDL was modified by different treatments: by incubation with SMase (10 U/g apoB) for $2 \mathrm{~h}$ at $37^{\circ} \mathrm{C}$ to increase its Cer concentration (SMase-LDL); by incubation with NEFA (NEFA-LDL) [28]; and by incubation with Sph-enriched liposomes $(10 \mu \mathrm{M})$ (Sph-LDL), as described for Cer-enriched liposomes [22]. The enrichment in these compounds was assessed by thin-layer chromatography, and the aggregation level of modified LDL was evaluated by gradient gel electrophoresis, as previously described [22].

Total LDL was fractionated in LDL(+) and LDL(-) by preparative anion-exchange chromatography in an ÄKTA-FPLC system (GE Healthcare) [37]. LDL fractions were concentrated with Amicon 
centrifugal filters (Merck Millipore). LDL(+) and LDL(-) compositions were determined in a Cobas ${ }^{\circledR}$ 501 autoanalyzer, including total cholesterol, triglyceride, apoB (Roche, Basel, Switzerland), NEFA, and phospholipid (Wako, Neuss, Germany).

\subsection{Lipidomic Analysis}

Baseline levels of sphingolipids in LDL(+) and LDL(-) were evaluated by liquid chromatographymass spectrometry (LC-SM) in the CIBERDEM-Metabolomics Platform of Universitat Rovira i Virgili. First, a lipid extraction of LDL samples (32 $\mu \mathrm{g}$ apoB) was performed. LDLs were lyophilized and resuspended in $220 \mu \mathrm{L}$ of methanol $(\mathrm{MeOH})$ followed by vortexing. Then, $440 \mu \mathrm{L}$ of dichloromethane were added followed by vortexing. Afterwards, $140 \mu \mathrm{L}$ of ultrapure water was added, vortexed, and stored at room temperature for $20 \mathrm{~min}$. The solution was centrifuged for $10 \mathrm{~min}$ at 14,500 rpm (4 ${ }^{\circ} \mathrm{C}$ ). Next, $400 \mu \mathrm{L}$ of the organic phase was collected and evaporated to dryness with $\mathrm{N}_{2}$. Samples were reconstituted in $150 \mu \mathrm{L}$ of $\mathrm{MeOH}$ :toluene (9:1) and transferred to LC-MS vials. Lipid extracts $(5 \mu \mathrm{L})$ were injected in a Ultra High Perfomance Liquid Chromatography system (1290 Agilent, Santa Clara, CA, USA) coupled to a triple quadrupole (QqQ) mass spectrometer (6490 Agilent Technologies) operated in positive electrospray ionization (ESI+) mode. The instrument was set to acquire in multiple reaction monitoring mode. Lipids were separated using C18-RP (ACQUITY UPLC BEH $2.1 \times 150 \mathrm{~mm}$, $1.7 \mu \mathrm{m}$, Waters) chromatography at $65^{\circ} \mathrm{C}$ and at a flow rate of $0.4 \mathrm{~mL} / \mathrm{min}$. The solvent system was $\mathrm{A}=$ acetonitrile:water (60:40) in $10 \mathrm{mM}$ of ammonium formate, and B = isopropanol:acetonitrile (90:10) in $10 \mathrm{mM}$ of ammonium formate. The gradient elution started at $15 \% \mathrm{~B}$ and went to $30 \%$ from minute 0 to $2,48 \%$ B from minute 2 to $2.5,82 \%$ B from minute 2.5 to 11 , and $99 \%$ B from minute 11 to 11.5 . Quality control samples (QC) consisting of pooled samples were injected before the first study sample and then periodically after four study samples.

Cer 14:0, Cer 16:0, Cer 18:0, Cer 20:0, Cer 22:0, Cer 24:0, Sph 18:1, and S1P 18:1 relative concentrations were analyzed by this method. Total Cer was considered to be the sum of all the Cer species evaluated.

\subsection{Inflammatory Action on Monocytes}

Peripheral blood from volunteers was collected, and mononuclear cells were isolated by density gradient centrifugation using a density solution of $1.077 \mathrm{~kg} / \mathrm{L}$ (Lympholyte Cedarlane) [37]. Cells were seeded on 12-well plates $\left(2 \times 10^{6}\right.$ cells $\left./ \mathrm{mL}\right)$, and monocytes were separated from lymphocytes according to their adhesive properties. All the subjects gave their written informed consent, and the study was conducted after approval from the Institutional Ethics Committee of the Hospital Sant Pau.

To evaluate the role of sphingolipids in the inflammatory and apoptotic effects of LDL(-), several inhibitors were tested: chlorpromazine (CPZ) (Sigma) is an inhibitor of SMase activity; D-erythro-2-(N-myristoyl amino)-1-phenyl-1-propanol (MAPP) (Cayman) is an inhibitor of neutral/alkaline CDase activity; and N,N-dimethylsphingosine (DMS) (Cayman) is an inhibitor of Sph kinase. The effect of these inhibitors is summarized in Table 1.

Table 1. Inhibitors used in the study.

\begin{tabular}{ccccc}
\hline Inhibitor & Abbreviation & Enzyme & Substrate & Product \\
\hline $\begin{array}{c}\text { Chlorpromazine } \\
\text { D-erythro-2-(N-myristoyl }\end{array}$ & CPZ & SMase & SM & $\begin{array}{c}\text { Cer and } \\
\text { phosphorylcholine } \\
\text { amino)-1-phenyl-1-propanol } \\
N, N \text {-dimethylsphingosine }\end{array}$ \\
\hline
\end{tabular}

All the inhibitors were used at $10 \mu \mathrm{M}$. LDL(+) and LDL(-) were incubated in the presence or absence of these inhibitors for $20 \mathrm{~h}$; afterwards, they were dialyzed against RPMI 1640 medium (supplemented with $1 \%$ fetal calf serum, $2 \mathrm{mM}$ of L-glutamine, $0.1 \mathrm{U} / \mathrm{L}$ of streptomycin, and $0.1 \mathrm{U} / \mathrm{L}$ of penicillin), and filtered in sterile conditions. Then, monocytes were incubated for $20 \mathrm{~h}$ with 
these LDL subfractions (60 mg apoB/L) (pre-incubation conditions). Monocytes were also incubated simultaneously with inhibitors plus LDL(+) or LDL(-) $(60 \mathrm{mg}$ apoB/L) (co-incubation), and without LDL (blank or basal condition) in the presence or absence of inhibitors. All the incubations were performed in the above-mentioned RPMI 1640 medium. Cell supernatants were collected after $20 \mathrm{~h}$ to evaluate the release of IL1 $\beta$, IL6, IL10, and MCP1, using ELISA kits (eBioscience for all, except Diaclone for IL1 $\beta$ ), as described [38], and following the manufacturer's recommendations. Moreover, monocytes were collected to analyze apoptosis by flow cytometry.

\subsection{Apoptosis Assay}

Apoptosis assays were performed in monocytes incubated with LDL(-) plus MAPP or DMS, and with LDL(-) pre-incubated with CPZ (all inhibitors at $10 \mu \mathrm{M}$ ). Apoptosis was determined by an Annexin V-FITC, Apoptosis detection kit (eBioscience-Invitrogen, Carlsbad, CA, USA). Briefly, monocytes were collected and resuspended in $200 \mu \mathrm{L}$ of binding buffer (200,000 cells). Cell suspension was stained with Annexin V-FITC, which binds to phosphatidylserine, for $10 \mathrm{~min}$ in darkness at room temperature. Afterwards, cells were washed and resuspended in $190 \mu \mathrm{L}$ of binding buffer. After the addition of $10 \mu \mathrm{L}$ of propidium iodide (PI), the fluorescence on stained cells was measured by flow cytometry in a MACSQuant ${ }^{\circledR}$ Analyzer MQ10 (Milteny Biotech GmbH, Bergisch Gladbach, Germany) and analyzed using MACSQuant software (Milteny Biotech $\mathrm{GmbH})$. Non-stained cells ( $\left.\mathrm{A}^{-} \mathrm{PI}^{-}\right)$were considered to be viable cells, $\mathrm{A}^{+} \mathrm{PI}^{-}$was considered to indicate early apoptosis, double staining $\left(\mathrm{A}^{+} \mathrm{PI}^{+}\right)$ was considered to indicate late apoptosis, and $\mathrm{A}^{-} \mathrm{PI}^{+}$was considered to indicate necrotic cells. Total apoptosis is the sum of early and late apoptosis.

\subsection{Statistical Analyses}

Differences between groups were tested using the Wilcoxon signed-rank test for paired data. Results were expressed as mean \pm SD. A value of $p<0.05$ was considered to be statistically significant.

\section{Results}

\subsection{LDL(-) Has an Increased Content in Sph and Cer}

We aimed to evaluate the content of Cer, Sph, and S1P in LDL(+) and LDL(-) by mass spectrometry. Figure 1 shows that LDL( $(-)$ had a much higher content in Cer than LDL(+), as previously described by a less sensitive method [22]. In addition, LDL(-) showed a twofold content in Sph compared to LDL(+), whereas S1P was undetectable in both LDL subfractions.

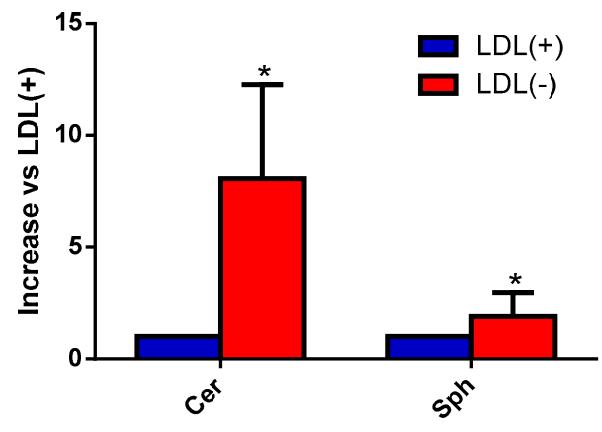

Figure 1. Sphingosine (Sph) and ceramide (Cer) basal content in low-density lipoprotein (LDL) subfractions. Native LDL(+) (blue bars) and negative LDL(-) (red bars) (32 $\mu \mathrm{g}$ of apolipoprotein B (apoB) were analyzed by mass spectrometry to evaluate the baseline levels of sphingolipids. Cer includes all the Cer species that were evaluated. Results are expressed as a relative increase versus $\operatorname{LDL}(+)$, mean $\pm \operatorname{SD}(n=4),{ }^{*}$ vs. $\operatorname{LDL}(+), p<0.05$. 


\subsection{Sph and Cer Are Involved in the Cytokine Release Promoted by LDL(-) in Monocytes}

The role of Cer, Sph, and S1P in the cytokine release promoted by LDL(-) in monocytes was evaluated. For this purpose, specific inhibitors involved in the generation of the above-mentioned sphingolipids were used, respectively: chlorpromazine (CPZ) (SMase inhibitor), D-erythro-2( $N$-myristoyl amino)-1-phenyl-1-propanol (MAPP) (CDase inhibitor), and $N, N$-dimethylsphingosine (DMS) (Sph kinase inhibitor).

Inhibitors alone did not modify the release of cytokines compared to basal values in the absence of LDLs (data not shown).

In the presence of LDL(-), the addition of DMS did not alter the release of monocyte chemoattractant protein 1 (MCP1) and interleukin (IL)-6 promoted by LDL(-), thereby suggesting that S1P generation was not involved in the inflammatory action of this lipoprotein (Figure 2). By contrast, the addition of CPZ inhibited the release of these cytokines induced by LDL(-).
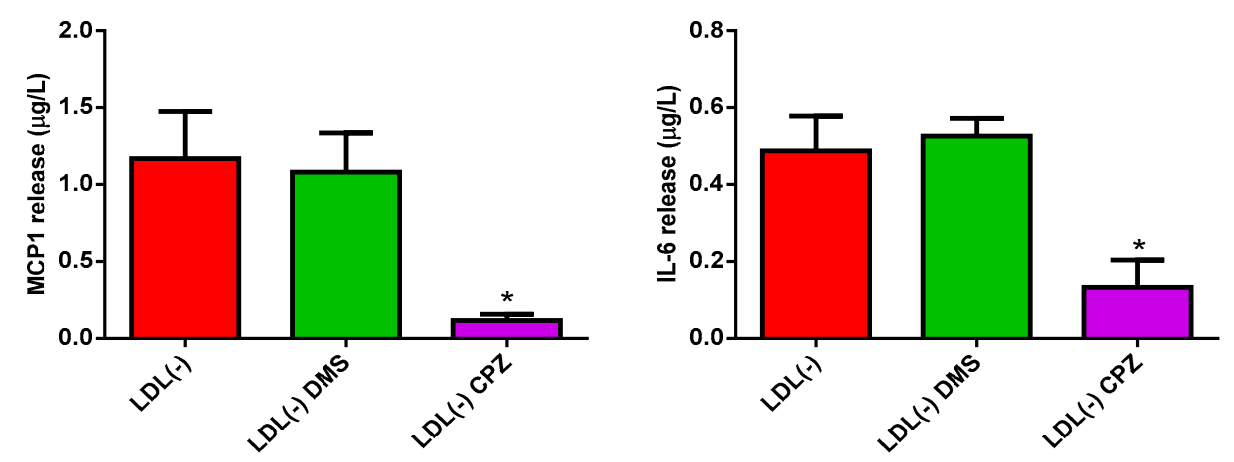

Figure 2. MCP1 and interleukin-6 (IL-6) release promoted by LDL(-) in human monocytes in the presence or absence of DMS and chlorpromazine CPZ. Monocytes were incubated with LDL(-) (60 $\mathrm{mg}$ apoB/L) in the absence (red bars) or presence of DMS (green bars) and CPZ (purple bars), both at $10 \mu \mathrm{M}$. After 20 hours of incubation, cytokine release was measured by ELISA. Results are expressed as mean $\pm \operatorname{SD}(n=5),{ }^{*}$ vs. absence of inhibitor, $p<0.05$.

Finally, the addition of MAPP decreased MCP1 and IL-6 release induced by LDL(-) as well as that of IL-1 $\beta$ and IL-10, in a concentration-dependent manner (Figure 3). However, the effect promoted by LDL(+) was not modified by the addition of any inhibitor (in the case of MAPP, inhibition lower than 5\%). Taken together, the results indicate that both Sph and Cer contribute to LDL(-)-induced inflammation in human monocytes.

To confirm the role of increased Sph and Cer content in LDL on the inflammatory response of monocytes, we conducted experiments in which LDL(+) was enriched "in vitro" with these compounds by incubation with liposomes containing Sph or by SMase lipolysis, respectively. Supplementary Figure S1 shows the enrichment of Sph and Cer in these "in vitro"-modified LDLs and the effect of such modification in the aggregation of LDL particles. The increase in Sph and Cer was 1.5-fold and sixfold, respectively, as calculated by densitometry versus unmodified LDL. Figure 4 shows that compared to unmodified LDL, LDL enriched with Sph (Sph-LDL), and LDL treated with SMase (SMase-LDL) induced the release of IL-6, as a representative cytokine induced by LDL(-). 

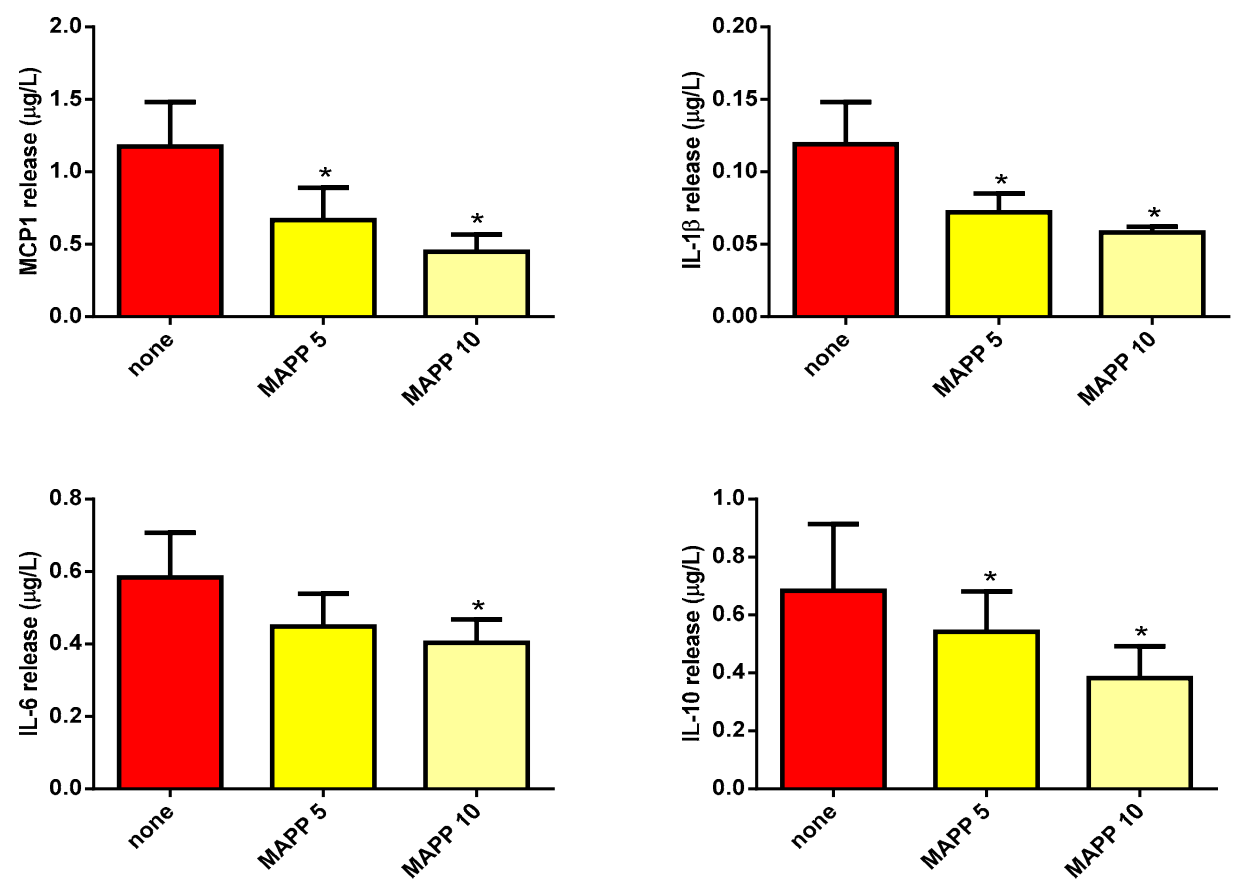

Figure 3. MCP1, IL-1 $\beta$, IL-6, and IL-10 release promoted by LDL(-) in human monocytes in the presence or absence of MAPP. Monocytes were incubated with LDL(-) (60 mg apoB/L) in the absence of MAPP (red bars) or in the presence of MAPP at $5 \mu \mathrm{M}$ (yellow bars) and $10 \mu \mathrm{M}$ (light yellow bars). After 20 hours of incubation, cytokine release was measured by ELISA. Results are expressed as mean $\pm \mathrm{SD}(n=5),{ }^{*}$ vs. the absence of inhibitor, $p<0.05$.

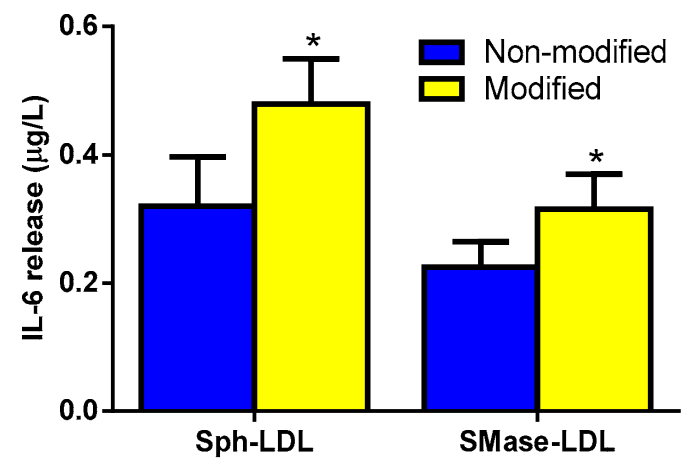

Figure 4. IL-6 release promoted by "in vitro" modified LDL. Monocytes were incubated with unmodified LDL (blue bars) or modified LDL (yellow bars), both at $60 \mathrm{mg}$ apoB/L. LDL was modified by incubation with Sph-enriched liposomes (at 0 and $10 \mu \mathrm{M}$ ) and by sphingomyelinase (SMase) treatment (at 0 and $10 \mathrm{U}$ enzyme/g apoB). After 20 hours of incubation, cytokine release was measured by ELISA. Results are expressed as $\mu \mathrm{g} / \mathrm{L}$ and as mean $\pm \mathrm{SD}(n=6),{ }^{*}$ vs. unmodified LDL, $p<0.05$.

Next, we sought to determine whether the inhibition induced by MAPP and CPZ on cytokine release was due to a direct effect on LDL(-) or was a consequence of an effect on cell enzymes. For this purpose, LDL(-) was pre-incubated with MAPP or CPZ, and then dyalized to remove excess inhibitor prior to its addition to monocytes. This effect was compared to that promoted when monocytes were incubated simultaneously with LDL(-) and an inhibitor (co-incubation). Figure 5 shows IL-6 release, as a representative cytokine induced by LDL(-). No significant difference was observed when using MAPP between pre-incubation and co-incubation. By contrast, CPZ exerted a more effective inhibitory effect in co-incubation than in pre-incubation conditions. These observations indicate that $\mathrm{CPZ}$ has a direct effect on cells, perhaps by inhibiting cell SMase. 


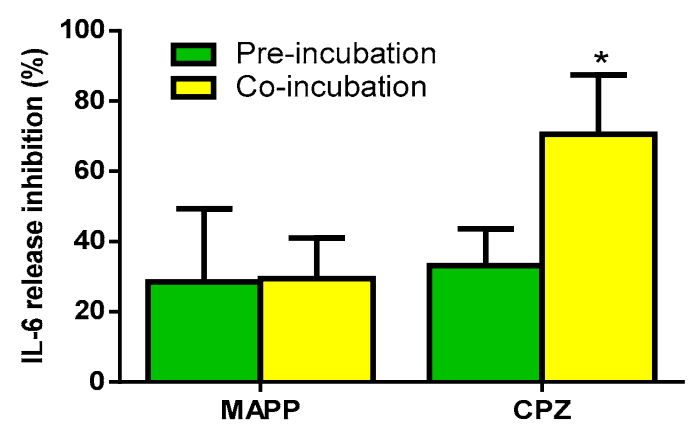

Figure 5. IL-6 release promoted by LDL(-) in pre-incubation or co-incubation with MAPP and CPZ. Monocytes were incubated with LDL(-) (60 mg apoB/L), which was pre-incubated (green bars) or co-incubated (yellow bars) with the inhibitors MAPP and CPZ, both at $10 \mu \mathrm{M}$. After $20 \mathrm{~h}$ of incubation, cytokine release was measured by ELISA. Results are expressed as \% of inhibition versus LDL(-) in the absence of an inhibitor, mean $\pm \operatorname{SD}(n=5),{ }^{*}$ vs. pre-incubation conditions, $p<0.05$.

\subsection{Cer Is Involved in LDL(-)-Induced Apoptosis}

Figure 6 shows that LDL(-) displayed a twofold increase in the total apoptotic effect on monocytes compared to LDL(+), which did not promote apoptosis. LDL(-) did not induce statistically significant differences in necrosis compared to LDL and the blank of cells.
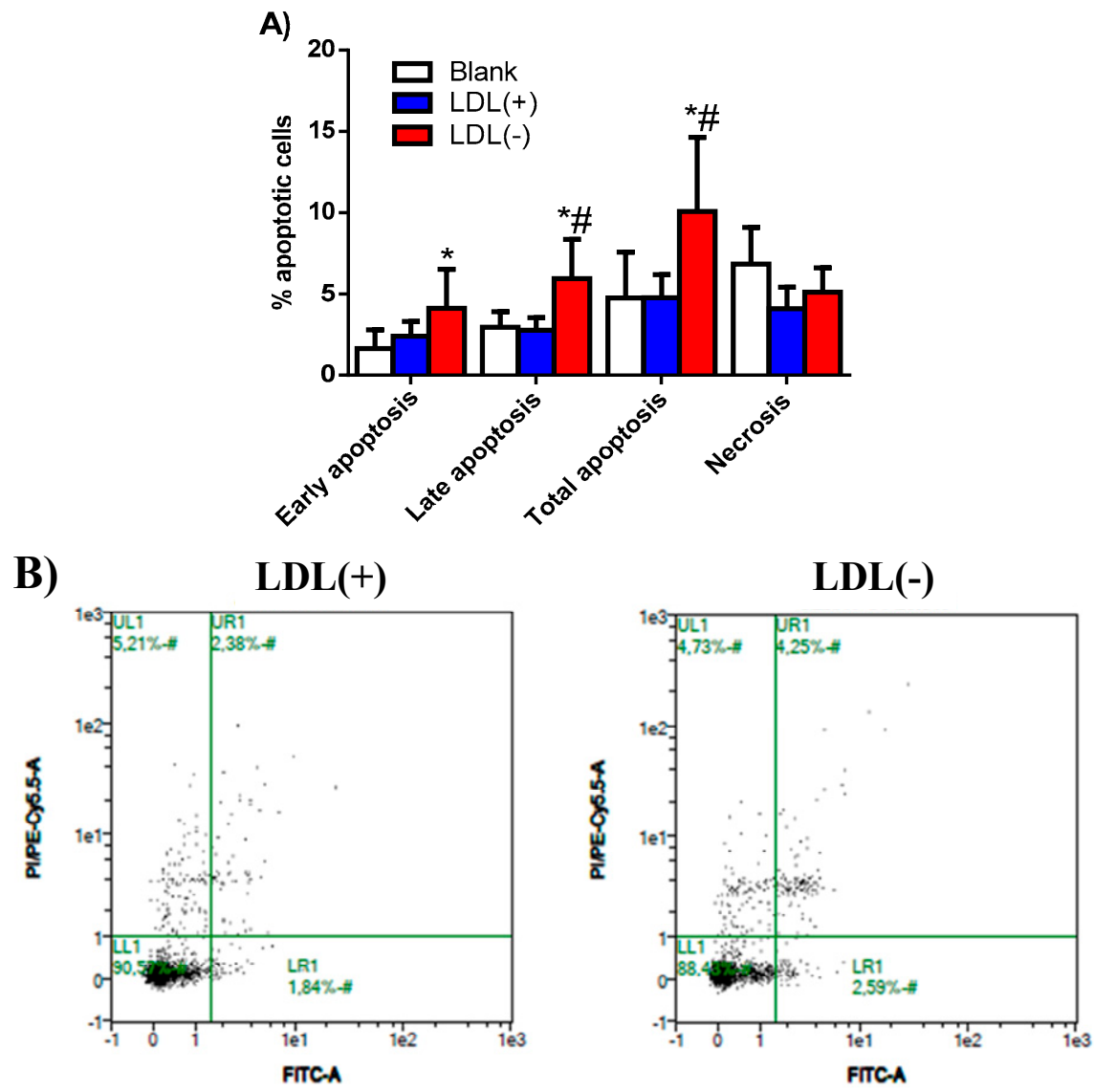

Figure 6. Apoptosis induced by $\operatorname{LDL}(+)$ and $\operatorname{LDL}(-)$ in human monocytes. (A) Early, late, and total apoptosis, and necrosis induced in basal conditions (blank) or by LDL(+) (blue bars) and LDL(-) (red bars) $(60 \mathrm{mg}$ apoB/L) were evaluated by flow cytometry. Results are expressed as percentage of apoptotic cells, mean $\pm \mathrm{SD}(n=6),{ }^{*}$ vs. blank cells, and \# vs. LDL $(+), p<0.05(\mathbf{B})$. Representative experiment showing the effect of LDL(+) (left panel) and LDL(-) (right panel) on human monocytes. 
The effect of enzyme inhibitors was also assayed in LDL(-)-induced apoptosis. Figure 7 shows that DMS enhanced the apoptotic effect of LDL(-), whereas CPZ decreased this effect, both in early and late apoptosis. These observations suggest that Cer is involved in LDL(-)-induced apoptosis, whereas S1P would tend to counteract this action. On the other hand, the presence of MAPP did not promote any significant effect, despite a slight tendency to increase late apoptosis.

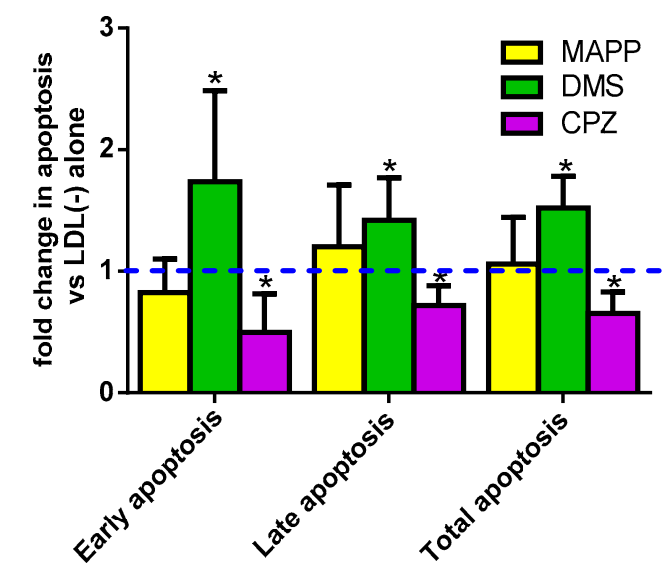

Figure 7. Apoptosis induced by LDL(-) in the presence of inhibitors. Early, late, and total apoptosis induced by LDL(-) (60 mg apoB/L) were evaluated in the absence or presence of MAPP (yellow bars), DMS (green bars), or CPZ (purple bars), all at $10 \mu \mathrm{M}$. Results are expressed as fold change in apoptosis versus $\operatorname{LDL}(-)$ in the absence of inhibitors, mean $\pm \operatorname{SD}(n=6),{ }^{*}$ vs. non-inhibitor, $p<0.05$.

However, MAPP and CPZ alone did not promote apoptosis in the absence of LDL(-), whereas DMS did (Supplementary Table S1).

LDL(+) was modified to increase its content in Cer, Sph, and NEFA, in order to reproduce the apoptotic behavior of LDL(-). Figure 8 shows that although NEFA-LDL and Sph-LDL promoted a slight non-significant apoptotic effect, the highest and statistically significant effect on total apoptosis was found when Cer content in LDL increased after SMase treatment.

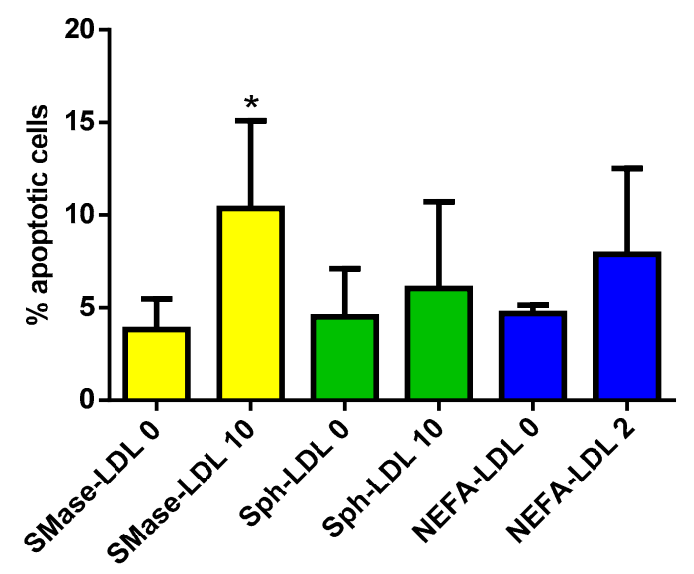

Figure 8. Total apoptosis induced by modified LDL(-). Total apoptosis induced by LDL modified by SMase treatment (yellow bars), Sph (green bars), or non-esterified fatty acid (NEFA) enrichment (blue bars), as described in the Methods section. Results are expressed as mean $\pm \operatorname{SD}(n=5),{ }^{*}$ vs. unmodified LDL, $p<0.05$.

\section{Discussion}

Sphingolipids and their metabolizing enzymes are described to be involved in atherogenesis. This study provides a novel contribution to understanding of the role of sphingolipids in inflammation and apoptosis, particularly focusing on the involvement of these metabolites in the atherogenic effects 
promoted by LDL(-) on monocytes. As determined by lipidomic analysis, LDL(-) had a higher content in Cer and Sph than LDL(+), and our findings show that the increase in these sphingolipids contributes to its inflammatory and apoptotic effects. Cer content in LDL(-) contributes to both LDL(-)-induced inflammatory and apoptotic effects, whereas Sph plays a more important role in its inflammatory effect. By contrast, S1P counteracts the apoptotic action exerted by LDL(-). These findings are summarized in Figure 9, which shows the effect of the inhibitors used in the study on sphingolipid generation and the ensuing LDL(-)-induced inflammatory and apoptotic actions.

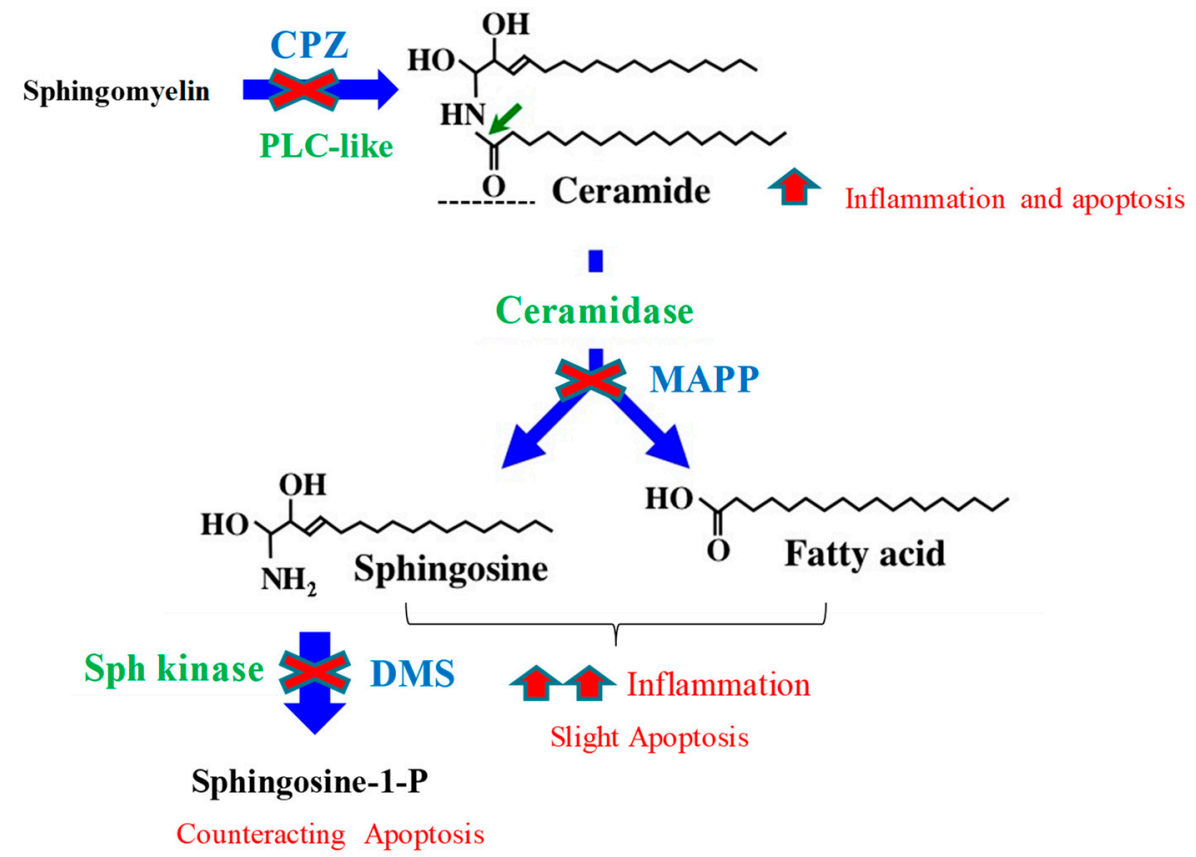

Figure 9. Effect of the inhibitors used in the study on sphingolipid generation and on LDL(-)-induced inflammatory and apoptotic actions. CPZ blocks sphingomyelin degradation, thereby decreasing Cer content and LDL(-)-induced cytokine release and apoptosis. MAPP blocks Cer degradation, leading to a decrease in Sph and NEFA generation, which seems to develop an essential role in inflammation, but a likely minor role in apoptosis. DMS blocks sphingosine-1-phosphate (S1P) generation, thus leading to an increase in the apoptotic effect of $\operatorname{LDL}(-)$.

The increased Cer content in LDL(-) was previously determined by thin layer chromatography, which is a much less sensitive method [22]. The current study corroborated this observation and also showed a higher content of Sph in LDL(-) compared to LDL(+). On the other hand, we found undetectable basal levels of S1P in both LDL fractions. Other authors have detected very low levels of S1P in LDL [21,39]. According to Hammad et al., the distribution of S1P in plasma lipoproteins was of $94.94 \%$ in HDL and only $3.73 \%$ in LDL. Compared to that study, the absence of S1P in our LDL subfractions could be due to different experimental conditions, such as the range of isolation of LDL and the lower concentration of the sample.

The inflammatory action of LDL(-) on monocytes was reported to be promoted, in part, by its increased content in NEFA and Cer [22,28]. The increased Cer content was proposed to be generated by the SMase activity ascribed to LDL(-) [22]. This hypothesis is supported by the current data, with the use of the SMase inhibitor CPZ, which inhibits the cytokine release promoted by LDL(-) in monocytes. Since CPZ blocks the degradation of sphingomyelin to yield Cer, it probably also blocks the generation of sphingolipids derived from Cer hydrolysis. In fact, $\mathrm{CPZ}$ was the inhibitor that promoted the greatest decrease in inflammation and apoptosis. The effect of $\mathrm{CPZ}$ was greater in co-incubation experiments with monocytes and LDL(-) added simultaneously, thereby suggesting that CPZ could block not only the intrinsic SMase activity in LDL(-), but also cell enzymatic activity. 
The role of Sph in inflammation is largely unknown, although it has been reported that it induces inflammasome activation, leading to IL-1 $\beta$ induction in macrophages [8]. The current study shows that Sph contributes to LDL(-)-induced inflammation in monocytes. This is suggested by the inhibition exerted by MAPP on the cytokine release promoted by LDL(-). However, MAPP is a CDase inhibitor, which hinders Sph and NEFA generation; thus, its inhibitory effect could also be partly promoted by decreasing NEFA. The inflammatory role of Sph was also suggested by the effect promoted by Sph-LDL. LDL(+), modified to increase its Sph content 1.5 -fold, promoted cytokine release to a similar extent to that promoted by LDL(-). Notably, this artificial enrichment was similar to the increased content of Sph found in LDL(-). The higher Sph content in LDL(-) seems to be generated by a CDase-like activity, the origin of which could be cells or LDL(-) itself. The latter is suggested by the lack of difference on LDL(-)-induced cytokine release between pre-incubation and co-incubation with MAPP.

S1P can display both pro-inflammatory and anti-inflammatory effects, depending on the S1P receptor subtype expressed on cells [40]. However, LDL(-) did not exhibit an increased content in S1P, and DMS did not inhibit LDL(-)-induced cytokine release in monocytes. Therefore, our results reject the involvement of S1P in LDL(-)-induced inflammation.

LDL(-) exerts an apoptotic effect in endothelial cells [41,42] and macrophages [29], but its effect on monocytes had not yet been determined. Some observations made it feasible, because in this cell type, LDL(-) induces molecules related to apoptosis, such as caspase-1 [36], Fas [43], and p38 mitogen-activated protein kinase [38]. Regarding other modified LDLs, oxidized LDL exerts a pro-apoptotic effect in macrophages [31,44]. In monocytes, oxidized LDL can promote both pro-apoptotic and anti-apoptotic effects [45,46], and enzimatically modified LDL promotes apoptosis [47]. In the current study, LDL(-) exerted a slight apoptotic action on monocytes, which is rather lower than its effect found previously on endothelial cells. This could be because the studies of endothelial cells were performed with L5, which only includes the most negative subfraction of LDL(-), and because the highest number of apoptotic cells (more than 40\%) was only found when L5 was isolated from dyslipidemic patients [41,48,49]. By contrast, when it was isolated from control subjects, apoptosis was lower than $10 \%$ [41], which is a similar value to that found in the current study. In addition, in endothelial cells, the effect was mediated through lectin-like oxidized low-density lipoprotein receptor-1 which is a receptor that is barely expressed in monocytes. The induction of apoptosis could lead to different consequences, depending on the cell type, which is considered to have a pro-atherogenic effect in endothelial cells, whereas in monocytes, it could be hypothesized that it is a physiological control mechanism to decrease the accumulation of inflammatory cells.

Some molecules whose concentration is increased in LDL(-), such as LPC, Cer, and NEFA [22,50], have been shown to be involved in apoptosis [2,32,51]. The results of the current study strongly suggest that Cer content is mainly responsible for the apoptosis promoted by LDL(-) on monocytes, which is in agreement with that reported for endothelial cells [30]. First, the inhibition of SMase activity decreased LDL(-)-induced apoptotic activity on monocytes to an extent similar to that promoted by $\operatorname{LDL}(+)$; second, an increased Cer concentration in $\operatorname{LDL}(+)$, which was promoted by SMase treatment, induced apoptosis on monocytes at the same level as that promoted by LDL(-).

It has been reported that $\mathrm{Sph}$ is able to exert apoptotic effects $[9,10]$. In our experiments, Sph was not involved in the apoptotic effect promoted by LDL(-). This fact could be explained by the overlapping biological effects promoted by MAPP, altering the balance between pro-apoptotic and anti-apoptotic molecules owing to CDase inhibition, because MAPP may exert both an anti-apoptotic effect by decreasing Sph and NEFA content, and an apoptotic effect by increasing Cer content. However, the inhibition of S1P formation by the addition of DMS to monocytes increased LDL(-)-induced apoptosis. This observation is in agreement with the assertion that S1P has anti-apoptotic effects [14].

In summary, our data showed that the increased Cer content in LDL(-) contributed to its inflammatory and apoptotic effects on monocytes. Sph played a key role in inflammation, and S1P counteracted the apoptotic effect of LDL(-). The main limitation of our study is that it is an "in vitro" approximation, and it is difficult to evaluate the effect of the different sphingolipids separately, owing 
to the complexity of their metabolism and their interconversion. In addition, other factors remain to be examined in future studies, including the presence of enzymatic activities in LDL(-), yielding its increased content in Sph, and the content of this bioactive sphingolipid through incubation with monocytes in the presence or absence of enzyme inhibitors.

\section{Conclusions}

The current study provides new knowledge regarding the role of sphingolipids in mediating LDL(-)-induced inflammatory and apoptotic effects in monocytes. Our data suggest that the increased Cer content in LDL(-) contributes to both effects. Not only Cer, but also its hydrolysis product Sph, seems to play a key role in inflammation, and S1P counteracts the apoptotic effect of LDL(-).

Supplementary Materials: The following are available online at http://www.mdpi.com/2218-273X/9/8/300/s1, Figure S1: Modification of LDL by Sph enrichment and SMase treatment, Table S1: Apoptosis (\% apoptotic cells) induced in the absence (blank) or presence of inhibitors at $10 \mu \mathrm{M}(n=5),{ }^{*}$ vs. blank, $p<0.05$.

Author Contributions: S.B. conceived the original idea, designed the study, and wrote the manuscript, with support from N.P. N.P., M.E., and L.J. carried out the experiments. J.L.S.-Q. designed the study and supervised the findings of the work. All authors discussed the results and contributed to the final manuscript.

Funding: S.B. and J.L.S.-Q. are supported by grants FIS PI13/00364 and PI16/00471 from the Instituto de Salud Carlos III (co-financed by the European Regional Development Fund). J.L.S.-Q. is a member of CIBER of Diabetes and Metabolic Diseases (CIBERDEM, CB07/08/0016, an Instituto de Salud Carlos III Project). S.B., J.L.S.-Q., and N.P. are supported by grants from Fundacio La Marato TV3 project 158/U/2017, and are members of the Quality Research Group 2017-SGR-1149 from Generalitat de Catalunya. S.B., J.L.S.-Q., and N.P. are members of the Group of Vascular Biology from the Spanish Atherosclerosis Society.

Acknowledgments: The authors thank Bianca Pascale for her technical assistance in data acquisition and the CIBERDEM-Metabolomics Platform of Universitat Rovira i Virgili for the acquisition of LC-SM data.

Conflicts of Interest: The authors declare that they have no conflicts of interest

\section{References}

1. Maceyka, M.; Spiegel, S. Sphingolipid metabolites in inflammatory disease. Nature 2014, 510, 58-67. [CrossRef] [PubMed]

2. Edsfeldt, A.; Duner, P.; Stahlman, M.; Mollet, I.G.; Asciutto, G.; Grufman, H.; Nitulescu, M.; Persson, A.F.; Fisher, R.M.; Melander, O.; et al. Sphingolipids contribute to human atherosclerotic plaque inflammation. Arterioscler. Thromb. Vasc. Biol. 2016, 36, 1132-1140. [CrossRef] [PubMed]

3. Mao, C.; Obeid, L.M. Ceramidases: Regulators of cellular responses mediated by ceramide, sphingosine, and sphingosine-1-phosphate. Biochim. Biophys. Acta 2008, 1781, 424-434. [CrossRef] [PubMed]

4. Arana, L.; Gangoiti, P.; Ouro, A.; Trueba, M.; Gomez-Munoz, A. Ceramide and ceramide 1-phosphate in health and disease. Lipids Health Dis. 2010, 9, 15. [CrossRef] [PubMed]

5. Havulinna, A.S.; Sysi-Aho, M.; Hilvo, M.; Kauhanen, D.; Hurme, R.; Ekroos, K.; Salomaa, V.; Laaksonen, R. Circulating ceramides predict cardiovascular outcomes in the population-based finrisk 2002 cohort. Arterioscler. Thromb. Vasc. Biol. 2016, 36, 2424-2430. [CrossRef] [PubMed]

6. Meeusen, J.W.; Donato, L.J.; Bryant, S.C.; Baudhuin, L.M.; Berger, P.B.; Jaffe, A.S. Plasma ceramides. Arterioscler. Thromb. Vasc. Biol. 2018, 38, 1933-1939. [CrossRef] [PubMed]

7. Woodcock, J. Sphingosine and ceramide signalling in apoptosis. IUBMB Life 2006, 58, 462-466. [CrossRef]

8. Luheshi, N.M.; Giles, J.A.; Lopez-Castejon, G.; Brough, D. Sphingosine regulates the nlrp3-inflammasome and il-1beta release from macrophages. Eur. J. Immunol. 2012, 42, 716-725. [CrossRef]

9. Suzuki, E.; Handa, K.; Toledo, M.S.; Hakomori, S. Sphingosine-dependent apoptosis: A unified concept based on multiple mechanisms operating in concert. Proc. Natl. Acad. Sci. USA 2004, 101, 14788-14793. [CrossRef]

10. Monick, M.M.; Cameron, K.; Powers, L.S.; Butler, N.S.; McCoy, D.; Mallampalli, R.K.; Hunninghake, G.W. Sphingosine kinase mediates activation of extracellular signal-related kinase and akt by respiratory syncytial virus. Am. J. Respir. Cell Mol. Biol. 2004, 30, 844-852. [CrossRef] 
11. Muller, J.; von Bernstorff, W.; Heidecke, C.D.; Schulze, T. Differential s1p receptor profiles on m1- and m2-polarized macrophages affect macrophage cytokine production and migration. Biomed. Res. Int. 2017, 2017, 7584621. [CrossRef] [PubMed]

12. Maceyka, M.; Harikumar, K.B.; Milstien, S.; Spiegel, S. Sphingosine-1-phosphate signaling and its role in disease. Trends Cell Biol. 2012, 22, 50-60. [CrossRef] [PubMed]

13. Spiegel, S.; Milstien, S. Sphingosine-1-phosphate: An enigmatic signalling lipid. Nat. Rev. Mol. Cell Biol. 2003, 4, 397-407. [CrossRef] [PubMed]

14. Cuvillier, O.; Pirianov, G.; Kleuser, B.; Vanek, P.G.; Coso, O.A.; Gutkind, S.; Spiegel, S. Suppression of ceramide-mediated programmed cell death by sphingosine-1-phosphate. Nature 1996, 381, 800-803. [CrossRef] [PubMed]

15. Zhi, L.; Leung, B.P.; Melendez, A.J. Sphingosine kinase 1 regulates pro-inflammatory responses triggered by tnfalpha in primary human monocytes. J. Cell Physiol. 2006, 208, 109-115. [CrossRef] [PubMed]

16. Lai, W.Q.; Irwan, A.W.; Goh, H.H.; Howe, H.S.; Yu, D.T.; Valle-Onate, R.; McInnes, I.B.; Melendez, A.J.; Leung, B.P. Anti-inflammatory effects of sphingosine kinase modulation in inflammatory arthritis. J. Immunol. 2008, 181, 8010-8017. [CrossRef]

17. Jin, J.; Lu, Z.; Li, Y.; Ru, J.H.; Lopes-Virella, M.F.; Huang, Y. Lps and palmitate synergistically stimulate sphingosine kinase 1 and increase sphingosine 1 phosphate in raw264.7 macrophages. J. Leukoc. Biol. 2018, 104, 843-853. [CrossRef]

18. Ruiz, M.; Frej, C.; Holmer, A.; Guo, L.J.; Tran, S.; Dahlback, B. High-density lipoprotein-associated apolipoprotein $\mathrm{m}$ limits endothelial inflammation by delivering sphingosine-1-phosphate to the sphingosine-1-phosphate receptor 1. Arterioscler. Thromb. Vasc. Biol. 2017, 37, 118-129. [CrossRef]

19. Poti, F.; Simoni, M.; Nofer, J.R. Atheroprotective role of high-density lipoprotein (hdl)-associated sphingosine-1-phosphate (s1p). Cardiovasc. Res. 2014, 103, 395-404. [CrossRef]

20. Sattler, K.; Levkau, B. Sphingosine-1-phosphate as a mediator of high-density lipoprotein effects in cardiovascular protection. Cardiovasc. Res. 2009, 82, 201-211. [CrossRef]

21. Hammad, S.M.; Pierce, J.S.; Soodavar, F.; Smith, K.J.; Al Gadban, M.M.; Rembiesa, B.; Klein, R.L.; Hannun, Y.A.; Bielawski, J.; Bielawska, A. Blood sphingolipidomics in healthy humans: Impact of sample collection methodology. J. Lipid Res. 2010, 51, 3074-3087. [CrossRef] [PubMed]

22. Estruch, M.; Sanchez-Quesada, J.L.; Beloki, L.; Ordonez-Llanos, J.; Benitez, S. The induction of cytokine release in monocytes by electronegative low-density lipoprotein (ldl) is related to its higher ceramide content than native ldl. Int. J. Mol. Sci. 2013, 14, 2601-2616. [CrossRef] [PubMed]

23. Boon, J.; Hoy, A.J.; Stark, R.; Brown, R.D.; Meex, R.C.; Henstridge, D.C.; Schenk, S.; Meikle, P.J.; Horowitz, J.F.; Kingwell, B.A.; et al. Ceramides contained in ldl are elevated in type 2 diabetes and promote inflammation and skeletal muscle insulin resistance. Diabetes 2013, 62, 401-410. [CrossRef] [PubMed]

24. Estruch, M.; Sanchez-Quesada, J.L.; Ordonez Llanos, J.; Benitez, S. Electronegative ldl: A circulating modified ldl with a role in inflammation. Mediators Inflamm. 2013, 2013, 181324. [CrossRef] [PubMed]

25. Benitez, S.; Sanchez-Quesada, J.L.; Ribas, V.; Jorba, O.; Blanco-Vaca, F.; Gonzalez-Sastre, F.; Ordonez-Llanos, J. Platelet-activating factor acetylhydrolase is mainly associated with electronegative low-density lipoprotein subfraction. Circulation 2003, 108, 92-96. [CrossRef] [PubMed]

26. Bancells, C.; Benitez, S.; Villegas, S.; Jorba, O.; Ordonez-Llanos, J.; Sanchez-Quesada, J.L. Novel phospholipolytic activities associated with electronegative low-density lipoprotein are involved in increased self-aggregation. Biochemistry 2008, 47, 8186-8194. [CrossRef] [PubMed]

27. Estruch, M.; Sanchez-Quesada, J.L.; Ordonez-Llanos, J.; Benitez, S. Ceramide-enriched ldl induces cytokine release through tlr4 and cd14 in monocytes. Similarities with electronegative ldl. Clin. Investig. Arterioscler. 2014, 26, 131-137. [CrossRef]

28. Bancells, C.; Sanchez-Quesada, J.L.; Birkelund, R.; Ordonez-Llanos, J.; Benitez, S. Hdl and electronegative ldl exchange anti- and pro-inflammatory properties. J. Lipid Res. 2010, 51, 2947-2956. [CrossRef]

29. Pedrosa, A.M.; Faine, L.A.; Grosso, D.M.; de Las Heras, B.; Bosca, L.; Abdalla, D.S. Electronegative ldl induction of apoptosis in macrophages: Involvement of nrf2. Biochim. Biophys. Acta 2010, 1801, 430-437. [CrossRef]

30. Ke, L.Y.; Chan, H.C.; Chen, C.C.; Lu, J.; Marathe, G.K.; Chu, C.S.; Wang, C.Y.; Tung, Y.C.; McIntyre, T.M.; Yen, J.H.; et al. Enhanced sphingomyelinase activity contributes to the apoptotic capacity of electronegative low-density lipoprotein. J. Med. Chem. 2016, 59, 1032-1040. [CrossRef] 
31. Deigner, H.P.; Claus, R.; Bonaterra, G.A.; Gehrke, C.; Bibak, N.; Blaess, M.; Cantz, M.; Metz, J.; Kinscherf, R. Ceramide induces asmase expression: Implications for oxldl-induced apoptosis. FASEB J. 2001, 15, 807-814. [CrossRef]

32. Artwohl, M.; Roden, M.; Waldhausl, W.; Freudenthaler, A.; Baumgartner-Parzer, S.M. Free fatty acids trigger apoptosis and inhibit cell cycle progression in human vascular endothelial cells. FASEB J. 2004, 18, 146-148. [CrossRef] [PubMed]

33. Boyanovsky, B.; Karakashian, A.; King, K.; Giltiay, N.; Nikolova-Karakashian, M. Uptake and metabolism of low density lipoproteins with elevated ceramide content by human microvascular endothelial cells: Implications for the regulation of apoptosis. J. Biol. Chem. 2003, 278, 26992-26999. [CrossRef] [PubMed]

34. Dersch, K.; Ichijo, H.; Bhakdi, S.; Husmann, M. Fatty acids liberated from low-density lipoprotein trigger endothelial apoptosis via mitogen-activated protein kinases. Cell Death Differ. 2005, 12, 1107-1114. [CrossRef] [PubMed]

35. Loidl, A.; Claus, R.; Ingolic, E.; Deigner, H.P.; Hermetter, A. Role of ceramide in activation of stress-associated map kinases by minimally modified ldl in vascular smooth muscle cells. Biochim. Biophys. Acta 2004, 1690, 150-158. [CrossRef] [PubMed]

36. Estruch, M.; Rajamaki, K.; Sanchez-Quesada, J.L.; Kovanen, P.T.; Oorni, K.; Benitez, S.; Ordonez-Llanos, J. Electronegative ldl induces priming and inflammasome activation leading to il-1beta release in human monocytes and macrophages. Biochim. Biophys. Acta 2015, 1851, 1442-1449. [CrossRef] [PubMed]

37. Benitez, S.; Bancells, C.; Ordonez-Llanos, J.; Sanchez-Quesada, J.L. Pro-inflammatory action of ldl(-) on mononuclear cells is counteracted by increased il10 production. Biochim. Biophys. Acta 2007, 1771, 613-622. [CrossRef] [PubMed]

38. Estruch, M.; Sanchez-Quesada, J.L.; Ordonez-Llanos, J.; Benitez, S. Inflammatory intracellular pathways activated by electronegative ldl in monocytes. Biochim. Biophys. Acta 2016, 1861, 963-969. [CrossRef]

39. Damirin, A.; Tomura, H.; Komachi, M.; Liu, J.P.; Mogi, C.; Tobo, M.; Wang, J.Q.; Kimura, T.; Kuwabara, A.; Yamazaki, Y.; et al. Role of lipoprotein-associated lysophospholipids in migratory activity of coronary artery smooth muscle cells. Am. J. Physiol. Heart Circ. Physiol. 2007, 292, H2513-2522. [CrossRef]

40. Obinata, H.; Hla, T. Sphingosine 1-phosphate in coagulation and inflammation. Semin. Immunopathol. 2012, 34, 73-91. [CrossRef]

41. Yang, C.Y.; Chen, H.H.; Huang, M.T.; Raya, J.L.; Yang, J.H.; Chen, C.H.; Gaubatz, J.W.; Pownall, H.J.; Taylor, A.A.; Ballantyne, C.M.; et al. Pro-apoptotic low-density lipoprotein subfractions in type ii diabetes. Atherosclerosis 2007, 193, 283-291. [CrossRef]

42. Lu, J.; Yang, J.H.; Burns, A.R.; Chen, H.H.; Tang, D.; Walterscheid, J.P.; Suzuki, S.; Yang, C.Y.; Sawamura, T.; Chen, C.H. Mediation of electronegative low-density lipoprotein signaling by lox-1: A possible mechanism of endothelial apoptosis. Circ. Res. 2009, 104, 619-627. [CrossRef] [PubMed]

43. Bancells, C.; Sanchez-Quesada, J.L.; Birkelund, R.; Ordonez-Llanos, J.; Benitez, S. Electronegative ldl induces fas and modifies gene expression in mononuclear cells. Front. Biosci. (Elite Ed.) 2010, 2, 78-86. [PubMed]

44. Seimon, T.A.; Nadolski, M.J.; Liao, X.; Magallon, J.; Nguyen, M.; Feric, N.T.; Koschinsky, M.L.; Harkewicz, R.; Witztum, J.L.; Tsimikas, S.; et al. Atherogenic lipids and lipoproteins trigger cd36-tlr2-dependent apoptosis in macrophages undergoing endoplasmic reticulum stress. Cell Metab. 2010, 12, 467-482. [CrossRef] [PubMed]

45. Namgaladze, D.; Kollas, A.; Brune, B. Oxidized ldl attenuates apoptosis in monocytic cells by activating erk signaling. J. Lipid Res. 2008, 49, 58-65.

46. Baird, S.K.; Hampton, M.B.; Gieseg, S.P. Oxidized ldl triggers phosphatidylserine exposure in human monocyte cell lines by both caspase-dependent and -independent mechanisms. FEBS Lett. 2004, 578, 169-174.

47. Jostarndt, K.; Gellert, N.; Rubic, T.; Weber, C.; Kuhn, H.; Johansen, B.; Hrboticky, N.; Neuzil, J. Dissociation of apoptosis induction and cd36 upregulation by enzymatically modified low-density lipoprotein in monocytic cells. Biochem. Biophys. Res. Commun. 2002, 290, 988-993.

48. Lu, J.; Jiang, W.; Yang, J.H.; Chang, P.Y.; Walterscheid, J.P.; Chen, H.H.; Marcelli, M.; Tang, D.; Lee, Y.T.; Liao, W.S.; et al. Electronegative ldl impairs vascular endothelial cell integrity in diabetes by disrupting fibroblast growth factor 2 (fgf2) autoregulation. Diabetes 2008, 57, 158-166.

49. Chen, C.H.; Jiang, T.; Yang, J.H.; Jiang, W.; Lu, J.; Marathe, G.K.; Pownall, H.J.; Ballantyne, C.M.; McIntyre, T.M.; Henry, P.D.; et al. Low-density lipoprotein in hypercholesterolemic human plasma induces vascular endothelial cell apoptosis by inhibiting fibroblast growth factor 2 transcription. Circulation 2003, 107, 2102-2108. 
50. Benitez, S.; Camacho, M.; Arcelus, R.; Vila, L.; Bancells, C.; Ordonez-Llanos, J.; Sanchez-Quesada, J.L. Increased lysophosphatidylcholine and non-esterified fatty acid content in ldl induces chemokine release in endothelial cells. Relationship with electronegative ldl. Atherosclerosis 2004, 177, 299-305.

51. Carpenter, K.L.; Dennis, I.F.; Challis, I.R.; Osborn, D.P.; Macphee, C.H.; Leake, D.S.; Arends, M.J.; Mitchinson, M.J. Inhibition of lipoprotein-associated phospholipase a2 diminishes the death-inducing effects of oxidised ldl on human monocyte-macrophages. FEBS Lett. 2001, 505, 357-363.

(C) 2019 by the authors. Licensee MDPI, Basel, Switzerland. This article is an open access article distributed under the terms and conditions of the Creative Commons Attribution (CC BY) license (http://creativecommons.org/licenses/by/4.0/). 